\title{
Patient safety in dentistry: development of a candidate 'never event' list for primary care
}

\author{
I. Black ${ }^{1,2}$ and P. Bowie ${ }^{\star 1,3}$
}

\section{In brief}

Introduction The 'never event' concept is often used in secondary care and refers to an agreed list of patient safety incidents that 'should not happen if the necessary preventative measures are in place'. Such an intervention may raise awareness of patient safety issues and inform team learning and system improvements in primary care dentistry. Objective To identify and develop a candidate never event list for primary care dentistry. Methods A literature review, eight workshops with dental practitioners and a modified Delphi with 'expert' groups were used to identify and agree candidate never events. Results Twohundred and fifty dental practitioners suggested 507 never events, reduced to 27 distinct possibilities grouped across seven themes. Most frequently occurring themes were: 'checking medical history and prescribing' (119, 23.5\%) and 'infection control and decontamination' $(71,14 \%)$. 'Experts' endorsed nine candidate never event statements with one graded as 'extreme risk' (failure to check past medical history) and four as 'high risk' (for example, extracting wrong tooth). Conclusion Consensus on a preliminary list of never events was developed. This is the first known attempt to develop this approach and an important step in determining its value to patient safety. Further work is necessary to develop the utility of this method.

\section{Introduction}

Published evidence indicates that significant numbers of patients are unintentionally but avoidably harmed in different healthcare settings worldwide. ${ }^{1}$ For example, in the UK it is estimated around $10 \%$ of hospital patients may suffer some form of iatrogenic harm, ${ }^{2}$ while between $1-2 \%$ of patient consultations in general medical practice involve 'errors' which may lead to subsequent patient safety incidents. ${ }^{3}$ Efforts to study the safety problem in these settings, implement improvement programmes and educate the care workforce have been ongoing in the past decade. ${ }^{4}$

In comparison, related research, improvement and educational activity are lacking

${ }^{1}$ Assistant Director (Clinical Effectiveness), NHS Education for Scotland, Glasgow, UK and ${ }^{2}$ National Clinical Lead, Healthcare Improvement Scotland, Edinburgh, UK; ${ }^{3}$ Programme Director (Safety \& Improvement), NHS Education for Scotland, Glasgow, UK and ${ }^{4}$ Honorary Associate Professor, Institute of Health and Wellbeing, University of Glasgow, UK

${ }^{*}$ Correspondence to: Dr Paul Bowie

Email: paul.bowie@nes.scot.nhs.uk

Refereed Paper. Accepted 30 January 2017

DOI: $10.1038 /$ sj.bdj.2017.456 or severely limited in primary care dental settings internationally. ${ }^{5}$ Arguably the most insightful research to-date relates to the recent publication of a review of never and serious events in dentistry between 2005-2014 in England. ${ }^{6}$ Analysis of reported dental patient safety incidents using two national datasets $(\mathrm{N}=32,263)$, showed that $20 \%$ originated in primary care, including a very small number related to wrong site extraction/surgery (designated as a never event in NHS England).

To focus attention on the more serious patient harm incidents that can occur in acute hospitals (including 'near-miss' cases) the 'never event' approach has been developed as a clinical risk management strategy ${ }^{7}$ and is used in National Health Service (NHS) hospitals in the UK and in other secondary care systems worldwide to facilitate learning and improvement. ${ }^{8-10}$ Development work in this area is underway in general medical practice. ${ }^{11}$ Different but largely overlapping never event definitions exist. It can be defined as 'a serious, largely preventable patient safety incident that should not occur if the available preventable measures were implemented by healthcare workers, ${ }^{8}$ while the NHS England definition overlaps strongly but is more detailed and specific (Box 1). ${ }^{7}$ A never event example in the hospital context could be 'performing a surgical procedure on the wrong limb', while in general medical practice the 'failure to refer a patient to a hospital specialist when there is a strong clinical suspicion of cancer' is suggested as a never event. As of April 2015, 'wrong tooth extraction' was designated as a dental never event in NHS England. ${ }^{7}$ The aforementioned national dataset review includes data on a small number of dental 'never events' in mainly hospital settings, including wrong site surgery, although it is worth noting that no specific research has been undertaken to further consider and develop the concept more specifically as a means to aid learning and system improvement across dentistry.

The rationale for devising and implementing lists of 'never events' is multifaceted: they can increase awareness of highly important patient safety issues among the care workforce; organisations and care teams can be alerted to proactively mitigate or, where possible, eliminate the associated risks; and there is increased 
accountability to patients and the public in acknowledging and dealing with these types of serious incidents. ${ }^{11}$ Additionally, where a 'never event' does occur, it is expected that the incident is formally reported and is thoroughly investigated by care teams to direct local learning and improvement efforts. ${ }^{7}$ It should also be noted that never events do not per se constitute unlawful acts.

Given the potential benefits of the 'never event' approach, the growing recognition of the need for patient safety to be at the forefront of primary care delivery, ${ }^{12}$ and the dearth of related activity in general dental practice, ${ }^{5-6}$ preliminary work was undertaken to begin building consensus around a list of candidate 'never events. The aim of this study, therefore, was to engage with 'frontline' and 'expert' dental practitioners with an interest in patient safety to identify, develop and validate a candidate list of 'never events' for primary care dentistry. The underlying assumption is that this approach would have potential to raise awareness of patient safety concerns amongst, for example, dental teams, policymakers, educators, and clinical effectiveness advisers, and so inform local learning and improvement to minimise risks to both patients and practices.

\section{Methods}

\section{Study design}

A mixed methods study was undertaken which included a rapid review of empirical and grey literature, holding workshops ${ }^{13}$ with general dental practice staff, patient safetyinformed dental educators and leaders, and an experienced patient safety/human factors specialist to consider possible never events and build consensus in this area, including use of a modified Delphi group. ${ }^{14}$ The study was informed by ergonomic participatory design principles, ${ }^{15}$ that is, engaging directly with frontline groups in a co-design process because they have the greatest subject matter expertise.

\section{Data collection}

\section{Identification of potential never events}

Firstly, preliminary scoping review work was undertaken to identify the types of patient safety incidents (circumstances where a patient was unintentionally harmed or could have been harmed) that occur in general dental practice. Background information was available from the Dental Defence Union (DDU), ${ }^{16}$ the UK

Box 1 Comparison of definitions and criteria to be met in determining if patient safety incident can be judged as a never event in healthcare

In this study: ${ }^{1}$

'A Never Event is defined as '... a serious, largely preventable patient safety incident that should not occur if the available preventable measures were implemented in healthcare.' It was agreed that the following five conditions must all be met:

Is known to cause harm to a patient, or has the potential to do so AND

Is preventable by the healthcare professional, team or organisation AND

Can be clearly and precisely defined AND

Can be detected AND

Is not an unlawful act

In NHS England: ${ }^{7}$

- They are wholly preventable where guidance or safety recommendations provide strong systematic barriers

- They are available at a national level

- They implanted by healthcare workers

- Each never event has the potential to cause serious patient harm or death (however serious harm or death is not required)

- There is evidence that it has occurred in the past (ie, it is a known source of risk)

- It can be easily defined, identified and continually measured. This requirement helps minimise disputes around classification and ensures focus on learning and improved patient safety

General Dental Council (GDC), ${ }^{17}$ the Scottish Public Services Ombudsman (SPSO), ${ }^{18}$ and from submissions of Significant Event Analysis (SEA) reports to NHS Education for Scotland (the national body responsible for the education and training of NHS workforce) from general dental practitioners (GDPs) as part of continuing professional development arrangements.

A rapid search of published policy documents and empirical literature using relevant electronic databases (Medline, Embase, Cinahl, and Psych-Info from 1 January 1990 to 31 July 2016), with a focus on errors and harms associated with dental care was undertaken and updated as the study progressed, resulting in a small number of relevant publications being uncovered confirming the limited research undertaken in this area. The scoping review provided clues to the areas of dental practice where things may go wrong for patients and dentists.

Next, we introduced the 'never event' concept to frontline dental practitioners. Over a 9-month period (from April 2015) the lead author attended seven educational events for vocational dental practitioners and trainers across Scotland and used these forums as an opportunity to collect data on possible 'never events. Prior to this, in 2014 the data collection process was piloted at a national event (Scottish Dental Practice Based Research Network) which was attended by 80 mixed discipline delegates (for example, dental practitioners, national leaders, educators and researchers).
The aim of these events was to introduce and consider patient safety in a dental context. All participants were briefly introduced to the concept of 'never events' and previously published criteria ${ }^{11}$ used to determine a 'never event' in UK general medical practice (Box 1). They were asked to reflect on this within the dental context (on their own or as part of a small group), then identify up to eight serious patient safety incidents which could potentially fit these definitional criteria and list them on a short, pre-designed pro-forma, which were collated by IB.

\section{Data analysis}

All generated data were combined into a list of potential never events by IB. Obvious duplicates were removed and similarly related incidents were merged and reworded. A thematic analysis ${ }^{19}$ of data was undertaken independently by both authors (a dentist and national patient safety lead, and a patient safety researcher), who met to compare analyses, co-develop and refine potential never event categories and related themes on an iterative basis, with disagreements being resolved by discussion until consensus was achieved.

\section{Generation of never event statements by 'experts'}

Two half-day workshops were then held separately by the authors during April 2016 with different 'expert' groups $(\mathrm{N}=5$ and $\mathrm{N}=3)$ of experienced dentists and educators. Expertise was accorded based on professional experience 


\begin{tabular}{|c|c|c|c|c|c|}
\hline & \multicolumn{5}{|c|}{ Risk } \\
\hline \multirow{2}{*}{ Likelihood Score } & 1 & 2 & 3 & 4 & 5 \\
\hline & Rare & Unlikely & Possible & Likely & Almost certain \\
\hline \multirow{3}{*}{ Dental Consequence Score } & 1 & 2 & 3 & 4 & 5 \\
\hline & Negligible & Minor & Moderate & Major & Catastrophic \\
\hline & $\begin{array}{l}\text { Minimal upset or injury } \\
\text { requiring no/minimal } \\
\text { intervention or treatment }\end{array}$ & $\begin{array}{l}\text { Minor upset or injury } \\
\text { requiring some minor } \\
\text { intervention }\end{array}$ & $\begin{array}{l}\text { Moderate injury requiring } \\
\text { professional intervention }\end{array}$ & $\begin{array}{l}\text { Major injury or loss with } \\
\text { significant remedial work } \\
\text { required to restore }\end{array}$ & $\begin{array}{l}\text { Irreversible damage - no } \\
\text { potential to reverse, } \\
\text { restore or replace }\end{array}$ \\
\hline
\end{tabular}

Risk scoring example: likelihood score of $3 \times$ consequence score of $2=6$

Risk grading category: $(1-3)=$ low risk; $(4-6)=$ moderate risk; $(8-12)=$ high risk; $(15-25)=$ extreme risk

Table 2 Frequency of suggested never events by themed aspects of dental care $(\mathrm{N}=507)$

\begin{tabular}{|c|c|c|}
\hline $\begin{array}{l}\text { Themed aspects of } \\
\text { dental care }\end{array}$ & Types of possible 'never events' suggested & $\begin{array}{l}\text { Frequency of } \\
\text { suggestions by theme } \\
\mathrm{N}(\%)\end{array}$ \\
\hline \multirow{4}{*}{$\begin{array}{l}\text { Checking medical history } \\
\text { and prescribing }\end{array}$} & Failure to check past medical history & \multirow{4}{*}{$119(23.5)$} \\
\hline & Incorrect prescribing & \\
\hline & Extraction when INR not checked & \\
\hline & Extraction when on IV bisphosphonates & \\
\hline \multirow{5}{*}{$\begin{array}{l}\text { Infection control and } \\
\text { decontamination }\end{array}$} & Using dirty/unsterilised equipment & \multirow{5}{*}{$71(14.0)$} \\
\hline & Reuse of single use items. & \\
\hline & Poor infection control & \\
\hline & Not following correct hand hygiene procedures & \\
\hline & Patient contracts BBV & \\
\hline \multirow{4}{*}{$\begin{array}{l}\text { Emergency drugs and } \\
\text { equipment }\end{array}$} & Emergency drugs out of date & \multirow{4}{*}{$45(8.9)$} \\
\hline & Equipment out of date & \\
\hline & No up to date CPR training & \\
\hline & Defibrillator defective or not being checked & \\
\hline \multirow{3}{*}{$\begin{array}{l}\text { Extracting or restoring the } \\
\text { wrong tooth }\end{array}$} & Extracting wrong tooth & \multirow{3}{*}{$51(10.1)$} \\
\hline & Restoring wrong tooth & \\
\hline & Incorrect dental charting & \\
\hline \multirow{3}{*}{ Treating the wrong patient } & Wrong record for the patient & \multirow{3}{*}{$45(8.9)$} \\
\hline & Wrong patient invited into the surgery & \\
\hline & Fitting the wrong lab work & \\
\hline \multirow{3}{*}{$\begin{array}{l}\text { Inhalation or ingestion } \\
\text { of foreign objects or } \\
\text { substance }\end{array}$} & Inhalation of, or swallowing, crown or instrument & \multirow{3}{*}{$34(6.7)$} \\
\hline & Not using rubber dam & \\
\hline & Hypochloride incident & \\
\hline \multirow{4}{*}{$\begin{array}{l}\text { Record keeping and } \\
\text { referrals }\end{array}$} & Forgetting to writing up notes & \multirow{4}{*}{$32(6.3)$} \\
\hline & Not including enough information in a patient note. & \\
\hline & No record of soft tissue examination & \\
\hline & Failure to send a referral & \\
\hline
\end{tabular}

and interest in patient safety education and initiatives. Participants were introduced to the never event concept and a summary of the development work progress at that stage. They were then asked to review the never event categories generated from suggestions by frontline practitioners and agree if their current thematic grouping was logical. This led to further refinement of interdependent or closely related suggestions on the list. For example, it was agreed that 'prescribing antibiotic medication to a patient known to have a penicillin allergy' is dependent on a medical history being taken, updated and checked, which led to further modification of this theme. Similarly, it was also agreed 'that extraction when the INR was not checked' was also related to the patient's medical history and was considered suitable to add to that theme.

The never event definitional criteria ${ }^{11}$ was then applied by the expert groups to generate draft candidate never event statements. All five never event criteria were considered in turn by the groups for each potential never event to determine inclusion on the candidate list. Compliance with all five criteria was necessary for inclusion. There was in-depth debate over full compliance with some criteria, and this is noted when the results are presented.

Possible never events that may have been missed were also considered, but no new data were generated. Finally a first draft list of candidate never event statements for general dental practice was developed and agreed, which was then sent by electronic mail to all participants on two occasions after each workshop for further review, to enhance consensus and validation, and to allow more time for consideration of events that may meet the definitional criteria. Minor amendments to wording were made based on feedback received. 


\begin{tabular}{|c|c|c|c|c|c|c|}
\hline \multirow{2}{*}{ Possible never event } & \multicolumn{5}{|c|}{ Compliance with individual never event criterion } & \multirow{2}{*}{$\begin{array}{l}\text { Potential compliance } \\
\text { with all criteria }\end{array}$} \\
\hline & 1 & 2 & 3 & 4 & 5 & \\
\hline Failure to check PMH & Y & Y & Y & Y & Y & Y \\
\hline Inhaling or swallowing crown or instrument & Y & Y & Y & Y & Y & Y \\
\hline Restoring the wrong tooth & Y & Y & $Y^{*}$ & Y & Y & Y \\
\hline Extracting the wrong tooth & Y & Y & $Y^{*}$ & Y & Y & Y \\
\hline latrogenic damage to adjacent tooth & Y & $N^{*}$ & Y & $N^{*}$ & Y & N \\
\hline Allergic reaction due to not checking PMH & Y & Y & Y & Y & Y & Y \\
\hline Using dirty instruments & Y & Y & Y & $Y^{*}$ & Y & Y \\
\hline Delay in sending urgent referral & Y & Y & Y & Y & Y & Y \\
\hline Delay in sending routine referrals & $Y^{*}$ & Y & Y & Y & Y & Y \\
\hline Treating the wrong patient & Y & Y & Y & Y & Y & Y \\
\hline Oxygen and emergency drugs not available & Y & Y & Y & Y & Y & Y \\
\hline
\end{tabular}

\section{Application of modified NPSA Risk Matrix}

To help quantify and prioritise the clinical risk associated with each candidate never event, the National Patient Safety Agency (NPSA) Risk Matrix $^{20}$ (modified slightly by the authors from the original to reflect more relevant and realistic outcomes in the context of general dental practice) was also applied by the expert groups (Table 1). Each group was asked to consider the likelihood of individual events occurring and their potential consequences in terms of patient harm. A risk matrix score was also assigned to each candidate never event using the formula: 'likelihood (score) multiplied (x) by consequence (score)', with scores then matched across to the appropriate risk grading category (that is, extreme risk, high risk, moderate risk, low risk). The pattern of scoring and agreement of risk grading was consistent among both groups for the majority of events but a few required in-depth debate to reach group consensus.

\section{Results}

\section{Examples of potential never events} and related themes

A total of 250 dental practitioners suggested 507 individual never events at the outset. After review and modification of this list of suggestions by the authors this was reduced to 27 distinct types of possible never events (for example, incorrect

Table 4 Modified NPSA Risk Matrix score and grading category assigned to each candidate never event by the expert groups

\begin{tabular}{l|l|l} 
Possible never event & $\begin{array}{l}\text { Modified NPSA Risk } \\
\text { Matrix score }\end{array}$ & $\begin{array}{l}\text { Risk Matrix grading } \\
\text { category }\end{array}$ \\
\hline Failure to check past medical history (PMH) & 16 & Extreme risk \\
\hline Inhalation or swallowing of a crown or instrument & 12 & High risk \\
\hline Restoring wrong tooth & 9 & High risk \\
\hline Oxygen and or emergency drugs not available & 9 & High risk \\
\hline Allergic reaction due to not checking PMH & 9 & High risk \\
\hline Extracting wrong tooth & 8 & High risk \\
\hline latrogenic damage to adjacent tooth & 6 & Moderate risk \\
\hline Delay in routine referral & 6 & Moderate risk \\
\hline Delay in urgent referral & 5 & Moderate risk \\
\hline Using dirty instruments & 4 & Moderate risk \\
\hline Treating the wrong patient & 4 & Moderate risk \\
\hline
\end{tabular}

prescribing; not following correct hand hygiene procedures; and wrong patient invited into the surgery) which were grouped across seven different themes (for example, checking medical history and prescribing; extracting or restoring wrong tooth; and record keeping and referrals). The most frequently occurring themes were 'checking medical history and prescribing (119, $23.5 \%$ ), 'infection control and decontamination' $(71,14 \%)$, and 'extracting or restoring the wrong tooth (51 [10.1\%]) (Table 2).

\section{Compliance with never event definitional criteria}

Of the 27 possible types of never events generated from the initial suggestions, a total of eleven were fully compliant with each of the five criteria for determining inclusion as a candidate never event (Table 3). As noted, despite our reported consensus, there may be some debate over whether some included candidate never events actually met all necessary criteria, as others may judge this process differently. 


\begin{tabular}{|c|c|}
\hline Preliminary never event statements & $\begin{array}{l}\text { Contributory } \\
\text { points from Table } 2\end{array}$ \\
\hline $\begin{array}{l}\text { Prescribing a drug to a patient which has previously caused an adverse reaction or has potential to interact adversely with the patient's current } \\
\text { medication. }\end{array}$ & $1 \& 6$ \\
\hline Not updating or checking a patient's medical history prior to undertaking dental extractions. & 1 \\
\hline $\begin{array}{l}\text { Undertaking clinical procedures without taking adequate precautions to avoid potential for inhalation or ingestion of crowns or endodontic } \\
\text { instruments }\end{array}$ & 2 \\
\hline Undertaking clinical care without having identified that the patient notes correspond to the patient presenting. & $3 \& 4$ \\
\hline Omitting to check that treatment plans and radiographs concur with clinical findings before any treatment is commenced. & $3 \& 4$ \\
\hline Failing to check that all referrals are sent and correspondence received from other consultants are checked to confirm treatment plans. & $8 \& 9$ \\
\hline Carrying out decontamination procedures which are not in line with current guidance or inspection requirements. & 7 \\
\hline Using decontamination equipment which has not been tested or maintained to guidance or manufacturers requirements. & 7 \\
\hline No regular checking system applied to ensure oxygen cylinders are available and that all recommended drugs are available and in date & 11 \\
\hline
\end{tabular}

\section{Application of modified NPSA Risk Matrix score and grading}

A single candidate never event was graded as 'extreme risk' (failure to check past medical history), with four others graded as 'high risk' (for example, restoring wrong tooth) and the final six events categorised as 'moderate risk' (for example, delay in urgent referral and treating the wrong patient) (Table 4).

\section{Final validation of candidate never events}

A total of nine candidate never event statements related to diverse aspects of the primary care dental work system were generated, agreed and endorsed by the expert groups after intensive face-to-face and email discussion as part of the modified Delphi process to make each of them more specific, explicit and clearly defined (Table 5). For example, 'undertaking decontamination procedures which are not in line with current guidance or 'failing to check reports received from orthodontists, or other referrals, to confirm charting before undertaking extractions'.

\section{Discussion}

\section{Summary of main findings}

The study achieved its aim of beginning a consensus building process to develop and validate a preliminary list of candidate never events for primary care dentistry. Consensus was achieved on a list of nine candidate never events covering a range of potentially serious system wide issues, most of which relate to patient safety checking procedures. To our knowledge this is the first such attempt to explore the relevance of the 'never event' concept and undertake related development work in primary care dentistry. It is also one of a small number of dental studies with an explicit focus in terms of developing a tool to help improve patient safety related work practices and performance in this setting, potentially reducing risks to practitioners and practices alike.

\section{Strengths and limitations}

A key strength was using mixed methods, particularly in terms of taking a participatory design approach with frontline practitioners and involving those who are 'expert' in this area. The study was also strongly influenced by previous work ${ }^{11}$ in a similar clinical setting, which provides an element of methodological rigour. However, our literature review was non-systematic which means we may have potentially omitted studies of importance. Study bias is likely because the frontline practitioners we engaged may not be representative of the dental practitioner population. There are likely to be never events that participants did not identify, which is why we believe this is a preliminary development study. We did not engage with other members of the dental team (for example, nurses and practice managers), or academic safety researchers or patients, who may have different insights about never events. In the wider UK context, the study is also limited by its 'Scottish-centric' nature given, for example, the different policy and regulatory arrangements in NHS England with regard to never events. However, we would argue that the findings from this study are still useful in terms of informing learning and improvement strategies in all primary care dentistry settings. The NPSA risk matrix model was adapted for the dental setting for pragmatic reasons, but we did not test or validate these adaptations in a robust manner. Finally, we believe that the consensus building methods employed were sufficiently robust, but a more in-depth approach to validation with more diverse groups of participants, augmented by use of a suitable validity quantification technique, ${ }^{21}$ may have strengthened study rigour.

\section{Comparison with published literature}

A few of the generated safety issues (for example, emergency drugs and equipment and incorrect prescribing) compare favourably with similar never events developed for UK general medical practice, ${ }^{11}$ providing some external validation of these findings. Other study issues identified have previously been identified as potentially serious dental patient safety incidents. For example, wrong tooth extraction, ${ }^{6,16,22}$ inhalation and ingestion, adverse reactions, dealing with medical emergencies ${ }^{6}$ and the importance of checking medical histories. ${ }^{23}$

Limited evidence is published on the development of interventions to prevent wrong tooth extraction such as the use of checklists, ${ }^{23-26}$ clinical guidelines, ${ }^{27}$ a crew resource management initiative to improve team communication ${ }^{28}$ and an educational programme $e^{29}$ - although the focus of some of 
these studies is on outpatient settings but we can assume the principles may be transferable. Bailey et al..$^{23}$ have identified other potential interventions to minimise risks in the dental setting including through the use of incident reporting systems, electronic notes and trigger tools. However, evidence of the feasibility and effectiveness of these approaches is not yet reported.

Both Pemberton ${ }^{30}$ and Bailey ${ }^{31}$ highlight the importance of tools, reflective practice and risk management strategies for use by dental teams in making patient care safer. Arguably the never event concept and approach informs each of the four strategic areas proposed by Pemberton $^{30}$ for improving patient safety: 1) Identifying threats to patient safety by incident reporting; 2) Evaluating incidents and identifying best practice; 3) Communication and education about patient safety; and 4) Building a safety culture. As a first step, therefore, in developing an explicit patient safety agenda and programme of work the never event approach, with more testing and development, may have some merit in the primary care dental setting.

\section{Implications for dental policy, practice and research}

It is clear that dentistry is a late developer as far as patient safety awareness is concerned, presumably related to the assumption that dentists are less likely to cause serious harm resulting in death or permanent injury than medical or pharmacy colleagues. Unlike in other areas of the UK, there is no contractual or regulatory requirement in general dental services in NHS Scotland to report or record significant incidents or events related to clinical treatment or care. It must be assumed that, through the provision of complex restorative and surgical interventions on a daily basis, unintentional (and potentially preventable) harm does occur - the limited evidence base demon-

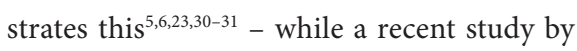
Pemberton and colleagues ${ }^{32}$ of NHS England never event data reported from hospitals and community trusts indicates that "wrong tooth/ teeth removed" is the most common "wrong site" event, "accounting for between $20 \%$ and $25 \%$ of wrong site surgery never events, and 6-9\% of all "never" events"' - a likely underestimate of the situation given that these data do not include wrong tooth extraction incidents from general dental practice. The findings from this study also strongly suggest knowledge amongst frontline practitioners about where things can or may go seriously wrong. The high volume of patients we see also provides multiplicity as far as risk of causing harm is concerned. Other than cases that come to the attention of bodies such as the defence organisations, the GDC or the SPSO there is little other evidence available.

The majority of study participants had never heard of the never event concept previously, although this approach has been in place for some time in medical settings (and is regularly referred to in the media). Despite that, they were consistent and certain as to what they believed constituted an event that should never happen in dental practice. Some aspects of the results were surprising. High profile never events in the acute sector are wrongside surgery or removing the wrong organ or limb, the analogous situation in dentistry being the extraction of the wrong tooth. This was not ranked particularly highly. It may be that dentists do not believe this could happen or that this event requires further reflection and clarification as it raises questions around grey areas related to accurate dental pain diagnosis.

Similarly treating the wrong patient was identified as having potentially significant consequences but was judged to be a very unlikely occurrence in the dental setting. A possible explanation could be the perception that dental practices are relatively small facilities and staff have local knowledge of many patients. In contrast, related acute hospital risks may be greater because of the interactions of high volumes of patients with multiple staff with limited local knowledge in largely one-off attendances. Understanding more about the actual prevalence of this phenomenon in the dental setting would obviously be important in terms of informing future risk management strategies.

Infection control and decontamination also featured frequently despite the fact that there is no compelling evidence of harm to patients. ${ }^{5,6,23,30,31}$ This may be explained by the highly emotive nature of this topic when infection control systems fail. Media coverage can be damaging for dentists and is an extremely emotive issue for patients.

Further validation of the study findings will be required amongst frontline practitioners and safety experts, which may well result in identification of other potentially serious harm events. The results suggest there are clear areas of practice with potential to harm patients, while the risk scoring method employed helped to suggest which should be prioritised.
Careful consideration will need to be given as to how the 'never event' concept might be introduced in dental practice, if it is judged to have possible benefit. Its value as a tool may be difficult to assess without baseline data on the frequency of never events occurring in the first place. Introducing processes to ensure these events are mitigated may be difficult if there is no perceived need, while the linkage between the approach and other initiatives to support patient safety and a just culture is underdeveloped in the dental setting. For example, most never event policies in healthcare have four main requirements: ${ }^{11}$

- Mandatory reporting of a specified incident when it occurs

- A rigorous, organisational-level investigation to determine why the incident occurred and to identify associated risk factors

- A responsibility to act on the findings and initiate changes to prevent a recurrence

- An apology to the patient concerned if appropriate.

In terms of mandatory reporting of never events and other safety incidents, some differences and potential confusions already exist between and within UK countries. For example, there is a professional responsibility on all dentists to report and learn from these incidents, ${ }^{33}$ but regulatory service expectations differ in Scotland where there is no such mandatory requirement in general dental services, unlike in England and Northern Ireland where the Care Quality Commission has a remit. ${ }^{34}$ Similarly, a myriad of different legal acts and regulatory bodies (for example, Mental Capacity Act, 2005; ${ }^{35}$ Control of Substances Hazardous to Health, 2002; ${ }^{36}$ Reporting of Injuries, Diseases and Dangerous Occurrences Regulations, 2013 ${ }^{37}$ ) also mandate the reporting of serious safety incidents in the workplace some of which may also cover the never events identified in this study, thus adding to the confusion. Overall, we also know that there is a limited incident reporting culture in primary care services in the UK and worldwide, which will need to be addressed if progress in learning about patient and staff safety is to be made. ${ }^{38}$

Clearly patient safety related research and improvement initiatives in dental practice are at an early embryonic stage, but policymakers in some countries recognise the potential need for action and a focus on this setting (for example, Scottish Patient Safety Programme in Primary Care $^{39}$ ). In terms of future safety 
research, studying the epidemiology of error and harm in this dental setting would arguably be a key priority, similar to that already achieved or underway in other areas of healthcare. ${ }^{1-3}$ More specific to this study, a further focus for research and evaluation would be necessary on the utility of the never event approach, that is, its acceptability and feasibility as an intervention, and related impact on practice team learning, system improvement and making care safer for patients and the dental team. Uncovering and addressing important issues that impact on staff safety and wellbeing, together with other quality of care issues, would also complement the never event approach. However, all of this will require recognition by national policymakers and research funders that there are patient and staff safety issues worthy of attention and a commitment to resource and support related initiatives.

\section{Conclusions}

Consensus on a candidate list of never events was developed based on the limited published evidence available, frontline practitioner experiences and expert input. This is the first known attempt to develop such an approach and an important starting point in determining the potential value of this concept as a patient safety initiative. The findings should be of wider interest in UK and international primary care dentals settings.

\section{Acknowledgements}

We offer sincere thanks to all vocational dental practitioners, trainers, researchers and leaders who kindly contributed to this study development.

\section{Ethical review}

Under UK 'Governance Arrangements for Research Ethics Committees', NHS ethical research committee review is not required for service evaluation or research which seeks to elicit the views, experiences, knowledge and contributions of healthcare professionals on a given subject area.

Funding

Study funding was provided by NHS Education for Scotland, Edinburgh, Scotland, UK.
1. The Health Foundation. Evidence scan. Levels of harm. London: The Health Foundation, 2011.

2. Vincent C, Neale G, Woloshynowych M. Adverse events in British hospitals: preliminary retrospective record review. BMJ 2001; 322: 517-519.

3. The Health Foundation. Evidence scan: levels of harm in primary care. London, The Health Foundation, 2011.

4. World Alliance for Patient Safety. Research for patient safety - better knowledge for safer care. Geneva: World Health Organization, 2008.

5. Bailey E, Tickle M, Campbell S. Patient safety in primary care dentistry: where are we now? Br Dent J 2014; 217: 339-344.

6. Thusu S, Panesar S, Bedi R. Patient safety in dentistry - state of play as revealed by a national database of errors. Br Dent J 2012; 213: E3.

7. NHS England. Revised Never Events Policy and Framework. NHS England. 2015; Available at https://www. england.nhs.uk/wp-content/uploads/2015/04/neverevnts-pol-framwrk-apr.pdf (accessed December 2015).

8. Milstein A. Ending extra payment for "never events" — stronger incentives for patients' safety. N Eng/ J Med 2009; 360: 2388-2390

9. National Quality Forum. Serious Reportable Events. Available online at http://www.qualityforum.org/ Topics/SREs/Serious_Reportable_Events.aspx (accessed January 2014).

10. Zahiri H R, Stromberg J, Skupsky $\mathrm{H}$ et al. Prevention of 3 'never events' in the operating room: fires, gossypiboma, and wrong-site surgery. Surg Innov 2011; 18: 55-60.

11. de Wet C., O'Donnell, C, Bowie P. Developing a preliminary 'never event' list for general practice using consensus-building methods. Br J Gen Pract 2014; 64: e159-e167.

12. Esmail A. Measuring and monitoring safety: a primary care perspective. The Health Foundation, 2013. Available online at http://www.health.org.uk/publication/ measuring-and-monitoring-safety-primary-care-perspective (accessed July 2016).

13. Jones J, Hunter D. Consensus methods for medical and health services research. BMJ1995; 311: 376-380.

14. Pill J. The Delphi method: substance, context, a critique and an annotated bibliography. Socio Econ Plan Sci 1971; 5: 57-71.

15. Hignett $S$, Wilson $J \mathrm{R}$, Morris W. Finding ergonomic solutions - participatory approaches. Occupational Med 2005; 55: 200-207.

16. Dental Defence Union. Rise in extraction error claims reports. London: DDU, 2013. Available online at http:/ www.theddu.com/press-centre/press-releases/rise-in-extraction-error-claims-reports-ddu (accessed July 2016).

17. General Dental Council. Annual Report and Accounts 2014. Available online at https://www.gdc-uk.org/api/ files $/ 17290 \% 20$ GDC $\% 20$ AR\%20ACC $\% 202014 \% 20$ R6\%20WEB\%20UD.pdf (accessed August 2016).

18. Scottish Public Services Ombudsman. Monthly News - July 2016. Available online at https://www.spso.org. uk/sites/spso/files/communications_material/commentary/2016/SPSOCommentary_July2016.pdf (accessed August 2016).

19. Saldana, Johnny. The Coding Manual for Qualitative Researchers. Thousand Oaks, California: Sage Publications, 2009.

20. National Patient Safety Agency. A risk matrix for risk managers. 2008. Available online at http://www. nrls.npsa.nhs.uk/EasySiteWeb/getresource.axd?As set $I D=60149 \&$ (accessed August 2016)
21. Lynn M R. Determination and quantification of content validity. Nurs Res 1986; 35: 382-385.

22. Peleg 0 , Givot $N$, Halamish-Shani T, Taicher S. Wrong tooth extraction: root cause analysis. Quintessence Int 2010; 41: 869-872.

23. Bailey E, Tickle M, Campbell S, O'Malley L. Systematic review of patient safety interventions in dentistry. $B M C$ Oral Health 2015; 15: 152

24. Saksena A, Pemberton M N, Shaw A, Dickson S, Ashley M P. Preventing wrong tooth extraction: experience in development and implementation of an outpatient safety checklist. Br Dent J 2014; 217: 357-362.

25. Perea-Perez B, Santiago-Saez A, Garcia-Marin F, Labajo Gonzalez E. Proposal for a 'surgical checklist' for ambulatory oral surgery. Int J Oral Maxillofac Surg 2011; 40: 949-954.

26. Beddis H P, Davies S J, Budenburg A, Horner K, Pemberton M N. Temporomandibular disorders, trismus and malignancy: development of a checklist to improve patient safety. Br Dent J 2014; 217: 351-355.

27. Lee J S, Curley A W. Prevention of wrong site tooth extraction: clinical guidelines. J Oral Maxillofac Surg 2007; 65: 1793-1799.

28. Pinsky H M, Taichman R S, Sarment D P. Adaptation of Airline Crew Resource Management Principles to Dentistry. JADA 2010; 141: 1010-1018.

29. Chang H H, Lee J J, Cheng S J et al. Effectiveness of an educational programme in reducing the incidence of wrong-site tooth extraction. Oral Surg Oral Med Oral Pathol Oral Radiol Endod 2004; 98: 288-294.

30. Pemberton MN. Developing patient safety in dentistry. Br Dent J 2014: 217: 335-337.

31. Bailey E. Contemporary views of dental practitioners on patient safety. Br Dent J 2015; 219: 535-540.

32. Pemberton M N, Ashley M P, Saksena A, Dickson S. Wrong tooth extraction: an examination of 'Never Event' data. Br J Oral Maxillofac Surg 2017; 55:187-188.

33. General Dental Council. Standards for the dental team. London: GDC, 2013

34. Care Quality Commission. How CQC regulates: primary care dental services. Available at http://www.cqc.org. uk/sites/default/files/20150611_dental_care_provider handbook.pdf (accessed December 2016).

35. Legislation.gov. Mental Capacity Act 2005. Available at http://www.legislation.gov.uk/ukpga/2005/9/contents (accessed December 2016).

36. Health and Safety Executive. Control of Substances Hazardous to Health. Available at http://www.hse.gov. uk/coshh/ (accessed December 2016).

37. Health and Safety Executive. Reporting of Injuries, Disease and Dangerous Occurrences Regulations 2013. Available at http://www.hse.gov.uk/riddor/ (accessed December 2016).

38. The Health Foundation. Measuring and monitoring safety: a primary care perspective. The Health Foundation: London, 2013.

39. Healthcare Improvement Scotland. Scottish Patient Safety Programme Primary Care. Patient. 2013. Available at http://www.healthcareimprovementscotland.org/ our_work/patient_safety/spsp/patient_safety_in_primary_care.aspx (accessed July 2016).

40. Department of Health. Governance Arrangements for Research Ethics Committees 2011. Available at http:// webarchive.nationalarchives.gov.uk/20130107105354/ http://www.dh.gov.uk/en/Publicationsandstatistics/Pub lications/PublicationsPolicyAndGuidance/DH_4005727 (accessed August 2016). 